\title{
Control of the Electric Load Simulator Using Fuzzy Multiresolution Wavelet Neural Network with Dynamic Compensation
}

\author{
Chao Wang, Yuanlong Hou, Rongzhong Liu, Qiang Gao, and Runmin Hou \\ School of Mechanical Engineering, Nanjing University of Science and Technology, Xiaolingwei 200, \\ Xuanwu, Nanjing, Jiangsu 210014, China
}

Correspondence should be addressed to Chao Wang; idiguatongxue@163.com

Received 10 June 2015; Revised 7 September 2015; Accepted 8 September 2015

Academic Editor: Vadim V. Silberschmidt

Copyright (C) 2016 Chao Wang et al. This is an open access article distributed under the Creative Commons Attribution License, which permits unrestricted use, distribution, and reproduction in any medium, provided the original work is properly cited.

\begin{abstract}
A fuzzy multiresolution wavelet neural network (FMWNN) controller with dynamic compensation (DC) is proposed to address the complexities of the electric load simulator (ELS). The FMWNN acts as a main torque tracking controller, which takes full advantage of the merits of an ideal sliding mode, fuzzy rules, and multiresolution WNN. The fuzzy algorithm is used to dynamically adjust the weights of the WNN and effectively accelerate the convergence rate. In addition, the DC controller is designed to greatly decrease the effect of the approximation error and guarantee the system stability in the sense of the Lyapunov theory. Finally, the proposed algorithms are carried out on the semiphysical simulation platform, the precision and superiority of which are comparatively verified based on the simulation results.
\end{abstract}

\section{Introduction}

During the past few years, load simulators are widely applied to the guns, aircrafts, ships, and so forth, which provide great help for simulating the real-time variable loads. On the basis of different load types, the load simulators can be mainly divided into electrohydraulic load simulators [1-7] and electrical load simulators (ELS) [5-7]. The former are usually used in the heavy load systems and the latter with the merits of high reliability, low noise and pollution, and easy maintenance are applied to the light load systems. However, the complexities of the ELS are significant, like friction, backlash, and self-coupling, which lead to the nonlinearity and uncertainty and being difficult to guarantee the control precision and stability. Easy and simple control algorithms (such as PID [5]) usually cannot meet the control requirements. In order to solve this problem, intelligent controllers are becoming important tools to achieve predominant control performance and robust fault-tolerant ability [8-12].

Neural networks (NN) have the advantages of parallel computation, approximation, self-learning, and fault tolerance $[13,14]$, which have been considerable interests in the nonlinear and uncertain control system [15-18]. However, the learning algorithms of the NN belong to an integraltype form, which can be trapped into the local minimum easily and make the low convergence rate of the network parameters. The excitation functions and weights of the networks play crucial roles in the approximation performance and adaptive neurons [14] and variable structures [18] are usually carried out to optimize the networks. As a common adaptive method, the wavelet neural networks (WNN) are mainly divided into the wavelet, scaling, and orthogonal wavelet function with different excitation functions [19-21]. The latter also called multiresolution WNN (MWNN) has the advantages of multiresolution analysis in time and frequency domain [21-23]. Since the real signals are represented by different resolution intervals, some features of the subseries can be more clearly reflected than the single resolution. Moreover, the weights of MWNN must be self-adaptive for improving the convergence rate, and the adjustments of weights follow the fuzzy rules proposed by many scholars [24-27]. Therefore, the fuzzy WNN emerges at the right moment and becomes a hot research topic [26, 27]. However, the adjustment of the range and convergence rate in $[26,27]$ 
is less than the fuzzy MWNN (FMWNN) comparatively. In order to further guarantee the stability and robustness, the sliding mode control (SMC) is used as an effective approach for nonlinear and uncertain control systems [28, 29], whereas the chattering problem in the SMC and the dead zone existing in the ELS lead to a finite steady state error [30, 31]. Many scholars adopt different kinds of compensation schemes, such as the mechanical structure adjustment [9], the friction and backlash disturbance model compensation [4, 31, 32], and the intelligent compensation algorithm $[18,33,34]$. However, the first two schemes only theoretically meet the control requirements. The intelligent compensation algorithm in accordance with the characteristics of the control system shows great superiority, which takes advantages of the input sliding mode and is introduced to dynamically decrease the effect of approximation error without exact models. In addition, the control system stability is guaranteed in the sense of the Lyapunov theory [18].

With the above-mentioned motivations, this paper proposes a fuzzy multiresolution wavelet neural network controller with dynamic compensation (DCFMWNN) to address the complexities of the ELS. Section 1 briefly introduces the background of the ELS and some control strategies. In Section 2, the thorny problem existing in the ELS is put forward, and an ideal controller is designed. In Section 3, the description of the proposed controller is clarified in combination with the ideal controller in detail. To verify the precision and superiority of the proposed algorithms, comparative simulation results are given out in Section 4. Finally, Section 5 concludes the paper.

\section{Problem Statement}

The AC permanent magnet synchronous motor (PMSM) is applied to the ELS for the gun control system. Though there are many merits in the PMSM, like the high ratio of the torque and inertia, the rapid and precise response, the uncertainties, and self-coupling disturbance result in negative impacts. In addition, the relationship between the control current and the output torque in PMSM is also not simple linear, which is different from the direct loading style. In combination with the working principle and practical working conditions of the ELS, the time-varying nonlinear system of the ELS can be simply described [35]:

$$
\ddot{x}(t)=f(\mathbf{x}, t)+g_{0} u(t)+d(t),
$$

where $\mathbf{x}=[x(t), \dot{x}(t)]$ and $u(t)$ are the state vector and the input of the control system, respectively; $f(\mathbf{x}, t)$ and $g_{0}$ are the dynamic variables, $f(\mathbf{x}, t)=-\left(B_{m} / J_{m}+\right.$ $\left.K_{T} K_{e} / J_{m} R_{m}\right) \dot{x}(t), g_{0}=K_{T} K_{a} / J_{m} R_{m}>0 ; B_{m}$ is the viscous frication coefficient of the motor; $J_{m}$ is the inertia constant of the motor; $R_{m}$ is the stator resistance of the motor; $K_{T}$ is the coefficient of the electromagnetic torque; $K_{e}$ is the coefficient of the counter electromotive force; $K_{a}$ is the amplifier gain; and $d(t)$ mainly includes the system external disturbance, which guarantees that $|d(t)|$ is less than $C_{0}$, where $C_{0}$ is a positive constant.

According to the existing problem, the control objective is to find a control law so that the state trajectory $x(t)$ can track a desired reference command $x_{d}(t)$; thus, a tracking error is defined as

$$
e_{1}(t)=x_{d}(t)-x(t)
$$

And a sliding surface is defined as follows:

$$
s(t)=\dot{e}_{1}(t)+k_{1} e_{1}(t),
$$

where $k_{1}$ is a positive constant. Consider the candidate Lyapunov function in the following form:

$$
V_{1}(t)=\frac{1}{2} e_{1}^{2}(t)+\frac{1}{2} s^{2}(t) .
$$

In combination with (2) and (3), differentiating (4) with respect to time obtains

$$
\begin{aligned}
\dot{V}_{1}(t)= & e_{1}(t) \dot{e}_{1}(t)+s(t) \dot{s}(t) \\
= & -k_{1} e_{1}^{2}(t)+s(t) e_{1}(t)+s(t) \dot{s}(t) \\
= & -k_{1} e_{1}^{2}(t)+s(t)\left[e_{1}(t)+k_{1} \dot{e}_{1}(t)+\ddot{x}_{d}(t)\right. \\
& \left.-f(\mathbf{x}, t)-g_{0} u(t)-d(t)\right] .
\end{aligned}
$$

If the system parameters in (1) are known, an ideal controller can be designed as [18]

$$
\begin{aligned}
& u_{\mathrm{IC}}^{*}(t) \\
& =\frac{\ddot{x}_{d}(t)-f(\mathbf{x}, t)-d(t)+e_{1}(t)+k_{1} \dot{e}_{1}(t)+k_{2} s(t)}{g_{0}},
\end{aligned}
$$

where $k_{2}$ is a positive constant. The following equation is obtained when $u(t)$ in (5) is replaced by $u_{\mathrm{IC}}^{*}(t)$ in $(6)$ :

$$
\dot{V}_{1}(t)=-k_{1} e_{1}^{2}(t)-k_{2} s^{2}(t) \leq 0 .
$$

Since $\dot{V}_{1}(t)$ is negative semidefinite, $V_{1}(t) \leq V_{1}(0)$. It implies that $e_{1}(t) \rightarrow 0$ and $s(t) \rightarrow 0$ are realized as $t \rightarrow \infty$, which are bounded [36]. Finally, the stability of the ideal controller can be guaranteed in the sense of the Lyapunov theory.

\section{Design of Fuzzy Multiresolution WNN with Dynamic Compensation}

The ideal controller $u_{\mathrm{IC}}^{*}(t)$ can asymptomatically stabilize the system. However, some nonlinear variables in $u_{\mathrm{IC}}^{*}(t)$ can not be obtained exactly, and the external disturbance $d(t)$ is uncertain in particular. In order to address this problem, the proposed DCFMWNN controller shown in Figure 1 is designed as

$$
u_{\mathrm{DCFMW}}=u_{\mathrm{FMW}}+u_{\mathrm{DC}},
$$

where $u_{\mathrm{FMW}}$ is the FMWNN controller and $u_{\mathrm{DC}}$ is a DC controller. The former is a main controller to mimic the ideal controller, and the latter based on a sliding surface compensates for the difference between the ideal controller and the former. The inputs of MWNN controller are torque errors and the derivation of torque errors and sliding surfaces. The input torque error is the difference between the reference command and torque sensor value. 


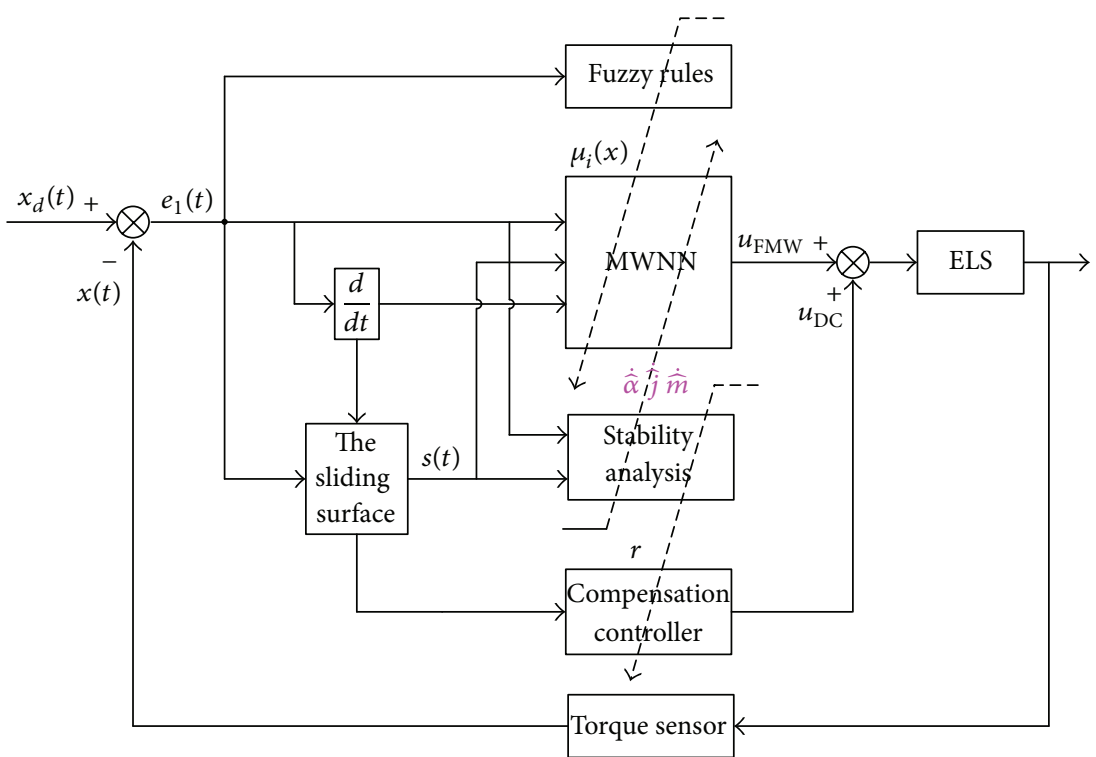

FIGURE 1: The block diagram of the DCFMWNN controller.

3.1. Wavelet Multiresolution Analysis. By virtue of different resolutions, the multiresolution analysis (MRA) can decompose a signal into components spanned by the scaling and wavelet basis functions. Any finite energy function can be represented as [21]

$$
L(x)=\sum_{m=-\infty}^{\infty} a_{j_{0}, m} \varphi_{j_{0}, m}(x)+\sum_{j=j_{0}}^{\infty} \sum_{m=-\infty}^{\infty} b_{j, m} \psi_{j, m}(x),
$$

where $\varphi_{j_{0}, m}(x)$ is the scaling function; $\psi_{j, m}(x)$ is the mother wavelet function; $a_{j_{0}, m}$ and $b_{j, m}$ are the expansion coefficients at the scaling levels $j_{0}$ and $j$, respectively. A higher or finer resolution function including more complete information of $L(x)$ is achieved with the addition of the scaling level $j$. The choice of the scaling and mother wavelet is application dependent; a popular mother wavelet is the Daubechies wavelet family defined as $\mathrm{db} X$, where $X$ represents the order. $\mathrm{db} X$ is orthogonal with regularity and compact support, which has been widely applied to various applications of wavelets.

3.2. Takagi-Sugeno-Kang Fuzzy System. With the advantages of easy computation and analysis, the fuzzy controllers with many adaptive and intelligent rules have been widely applied to complex nonlinear systems. Considering the multi-input single output (MISO) system, Takagi and Sugeno provide that the output of fuzzy rules is obtained by the linear combination of the inputs [37]. The following rules can be achieved [27]:

$$
\begin{gathered}
R_{i} \text { : IF } x_{1} \text { is } A_{1}^{i} \text { AND } x_{2} \text { is } A_{2}^{i} \cdots x_{n} \text { is } A_{n}^{i} \\
\text { THEN } y_{i}=h_{i 0}+h_{i 1} x_{1}+\cdots+h_{i n} x_{n} \quad k=1,2 \ldots, n,
\end{gathered}
$$

where $R_{i}$ is the $i$ th fuzzy rule; $h_{i k}$ is the link weight; $h_{i 0}$ is a constant; $x_{i}$ and $A_{k}^{i}$ represent the input torque error and fuzzy sets. Then, the system output with $D$ rules is

$$
y=\sum_{i=1}^{D}\left(h_{i 0}+h_{i 1} x_{1}+\cdots+h_{i n} x_{n}\right) \lambda_{i}(x),
$$

where $\lambda_{i}(x)$ denotes the basis function and usually is chosen as $\mu_{i}(x) / \sum_{i=1}^{D} \mu_{i}(x)$ and $D$ is the number of the fuzzy rules. And $\mu_{i}(x)=\exp \left(-\left(\left(x-c_{i k}\right)^{2} / \sigma_{i k}\right)^{2}\right)$ is the Gaussian membership function and $c_{i k}$ and $\sigma_{i k}$ are the center and variance of the membership function related to the $i$ th fuzzy rule, which are adjusted based on the gradient descent method.

3.3. Fuzzy Wavelet Multiresolution Analysis. The orthogonal wavelet function is usually used as the excitation function of neurons in the neural network, and the wavelet decomposition is used to analyse the data. The networks are trained step by step on the basis of multiresolution analysis. The output of FMWNN controller can be represented as

$$
\begin{aligned}
\Gamma(x) & =\sum_{m=m_{0}}^{D_{j_{0}}} a_{j_{0}, m} \varphi_{j_{0}, m}(x)+\sum_{j=j_{0}}^{J} \sum_{m=m_{0}}^{D_{j}} b_{j, m} \psi_{j, m}(x), \\
\varphi_{j, m} & =\frac{\varphi_{j, m}^{\prime} \mu_{k}\left(x_{i}\right)}{\sum_{k=1}^{D_{j_{0}}} \mu_{k}\left(x_{i}\right)} \\
& =\frac{\exp \left(-2^{j / 2}\left(x_{i}-m\right)^{2} / 2\right) \mu_{k}\left(x_{i}\right)}{\sum_{k=1}^{D_{j 0}} \mu_{k}\left(x_{i}\right)}, \\
\psi_{j, m} & =\frac{\psi_{j, m}^{\prime} \mu_{k}\left(x_{i}\right)}{\sum_{k=1}^{D_{j}} \mu_{k}\left(x_{i}\right)} \\
& =\frac{\left(1-x_{i}^{2}\right) \exp \left(-2^{j / 2}\left(x_{i}-m\right)^{2} / 2\right) \mu_{k}\left(x_{i}\right)}{\sum_{k=1}^{D_{j}} \mu_{k}\left(x_{i}\right)},
\end{aligned}
$$

where $i=1,2, \ldots, p$, and $j=j_{0}, j_{1}, \ldots, J . p$ and $J$ are the number of the inputs and locations on the grid at the resolution $j$, respectively [26]; $j$ and $m$ are the dilation and translation parameters, respectively; $\varphi_{j, m}$ and $\psi_{j, m}$ are the 


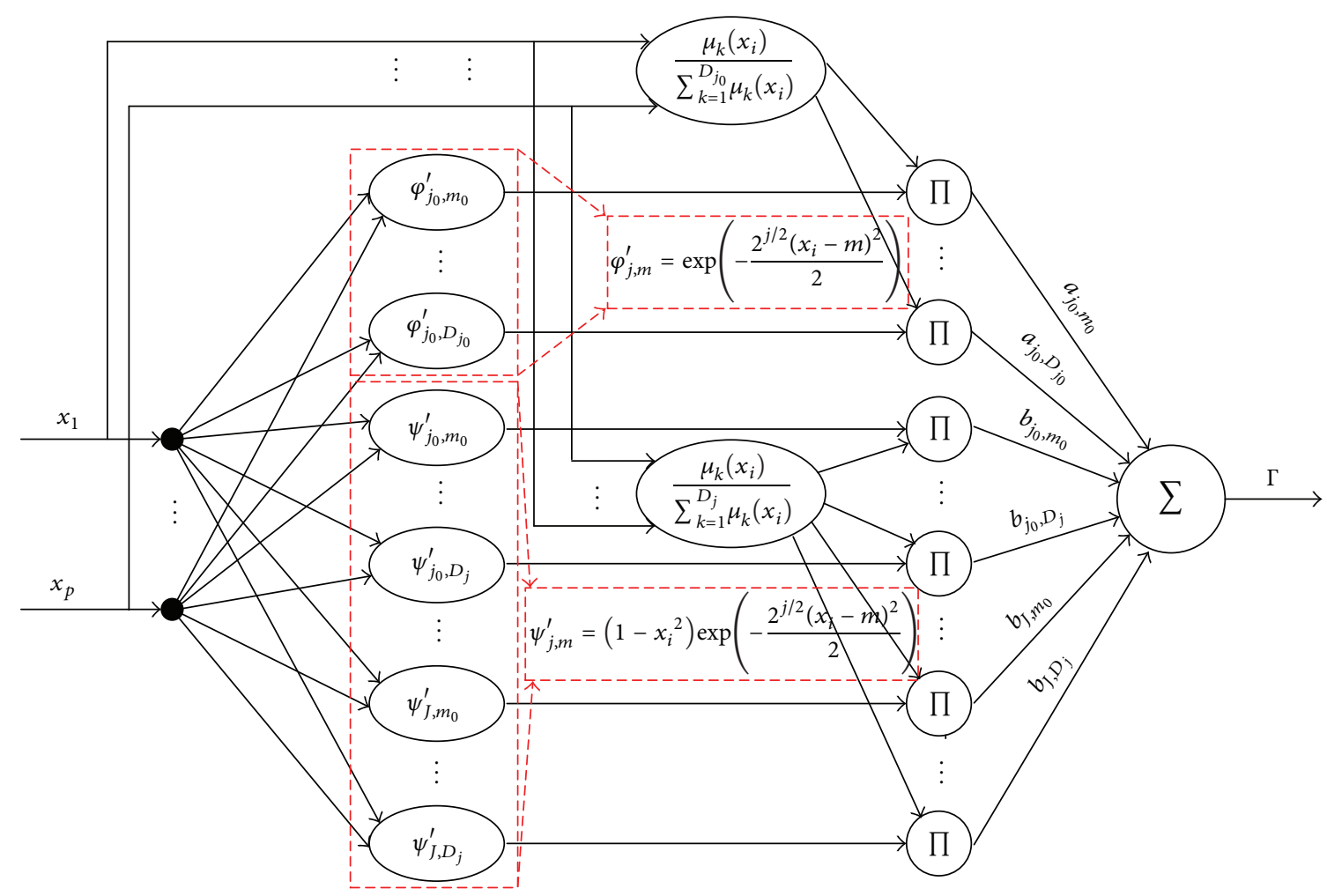

FIgURE 2: The structure of the FMWNN.

Gaussian scaling and wavelet functions, respectively. The coefficients $a_{j_{0}, m}$ and $b_{j, m}$ are derived based on a gradient descent algorithm and aim to minimize $s(t) \dot{s}(t)$ for achieving fast convergence [18], and the number of fuzzy rules depends on the summation bounds of $D_{j_{0}}$ and $D_{j}$ that can be determined from the time supports of both the output of FMWNN controller and the Gaussian scaling and wavelet functions. The higher level $J$ that adds finer resolution to the signal can be chosen as larger than $j_{0}$ as possible provided that the computational effort is not a concern, and several other criteria for the determination of $J$ are given in [22]. In combination with the characteristics of $\mathrm{db} X$ wavelets and simulation results, the lower level $j_{0}$ is defined as $-\operatorname{int}\left(\log _{2} N_{t}\right)$ and $X$ is set to be 2 , where $\operatorname{int}(\cdot)$ is the round-up integer with $2^{-j_{0}} \geq N_{t}>$ $2^{-j_{0}-1}$ and $N_{t}$ is the length of sampled data [23]. The MRA scaling can be set as $j_{0}=-14$ and $J=-8$, and $m_{0}, D_{j_{0}}$, and $D_{j}$ in (12) are chosen as $m_{0}=2-2 X, D_{j_{0}}=4$, and $D_{j}=$ $\operatorname{int}\left(2^{j} N_{t}\right)-1$. The proposed FMWNN is shown in Figure 2.

3.4. Dynamic Compensation Controller. According to the property of the universal function approximation, it implies that there exists an expansion of (6), which can approximate a nonlinear function $u_{\mathrm{FMW}}^{*}(t)$ as follows [38]:

$$
u_{\mathrm{IC}}^{*}(t)=u_{\mathrm{FMW}}^{*}(t)+\Delta=\boldsymbol{\alpha}^{* T} \Theta^{*}+\Delta,
$$

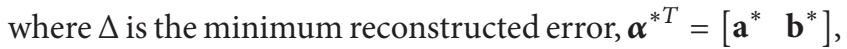
$\Theta^{* T}=\left[\boldsymbol{\varphi}^{*} \psi^{*}\right]$, and $\mathbf{a}^{*}, \mathbf{b}^{*}, \boldsymbol{\varphi}^{*}$, and $\psi^{*}$ are the ideal values, which can not be obtained precisely. Since these optimized parameters are unobtainable to guarantee best approximation, we adopt the online adaptive methods based on the offline learning. An online evaluation $\widehat{u}_{\text {FMW }}$ is used to estimate the optimized $u_{\mathrm{FMW}}^{*}(t)$, and (8) can be rewritten as

$$
u_{\mathrm{DCFMW}}=\widehat{u}_{\mathrm{FMW}}+u_{\mathrm{DC}}=\widehat{\boldsymbol{\alpha}}^{T} \widehat{\boldsymbol{\Theta}}+u_{\mathrm{DC}}
$$

where $\widehat{\boldsymbol{\alpha}}$ and $\widehat{\boldsymbol{\Theta}}$ are the estimation values of $\boldsymbol{\alpha}^{*}$ and $\boldsymbol{\Theta}^{*}$, respectively. An estimation error $\tilde{u}$ by subtracting (16) from (15) is defined as [18]

$$
\begin{aligned}
\widetilde{u} & =u_{\mathrm{IC}}^{*}-u_{\mathrm{DCFMW}}=\boldsymbol{\alpha}^{* T} \boldsymbol{\Theta}^{*}+\Delta-\widehat{\boldsymbol{\alpha}}^{T} \widehat{\boldsymbol{\Theta}}-u_{\mathrm{DC}} \\
& =\widetilde{\boldsymbol{\alpha}}^{T} \widetilde{\boldsymbol{\Theta}}+\widehat{\boldsymbol{\alpha}}^{T} \widetilde{\boldsymbol{\Theta}}+\widetilde{\boldsymbol{\alpha}}^{T} \widehat{\boldsymbol{\Theta}}+\Delta-u_{\mathrm{DC}},
\end{aligned}
$$

where $\widetilde{\boldsymbol{\alpha}}=\boldsymbol{\alpha}^{*}-\widehat{\boldsymbol{\alpha}}$ and $\widetilde{\boldsymbol{\Theta}}=\boldsymbol{\Theta}^{*}-\widehat{\boldsymbol{\Theta}}$. Moreover, the linearization technique is employed to transform the wavelet function into a partially linear form. The expansion of $\widetilde{\boldsymbol{\Theta}}$ in Taylor series can be obtained as [39]

$$
\widetilde{\Theta}=\left[\begin{array}{c}
\widetilde{\Theta}_{1} \\
\vdots \\
\widetilde{\Theta}_{i} \\
\vdots \\
\widetilde{\Theta}_{n a}
\end{array}\right]
$$




$$
\begin{aligned}
& =\left.\left[\begin{array}{c}
\left(\frac{\partial \widetilde{\Theta}_{1}}{\partial \mathbf{j}}\right) \\
\vdots \\
\left(\frac{\partial \widetilde{\Theta}_{i}}{\partial \mathbf{j}}\right) \\
\vdots \\
\left(\frac{\partial \widetilde{\Theta}_{n a}}{\partial \mathbf{j}}\right)
\end{array}\right]\right|_{j=\hat{j}}\left(\mathbf{j}^{*}-\widehat{\mathbf{j}}\right) \\
& \int_{0}^{T} s^{2}(t) d t \leq \frac{e^{2}(0)}{g_{0}^{2}}+\frac{s^{2}(0)}{g_{0}^{2}}+\frac{\widetilde{\boldsymbol{\alpha}}^{T}(0) \widetilde{\boldsymbol{\alpha}}(0)}{\eta_{1} g_{0}} \\
& +\frac{\widetilde{\mathbf{j}}^{T}(0) \tilde{\mathbf{j}}(0)}{\eta_{2} g_{0}}+\frac{\widetilde{\mathbf{m}}^{T}(0) \widetilde{\mathbf{m}}(0)}{\eta_{3} g_{0}} \\
& +\frac{r^{2}}{g_{0}^{2}} \int_{0}^{T} \Delta_{t}^{2}(t) d t
\end{aligned}
$$$$
+\left.\left[\begin{array}{c}
\left(\frac{\partial \widetilde{\Theta}_{1}}{\partial \mathbf{m}}\right) \\
\vdots \\
\left(\frac{\partial \widetilde{\Theta}_{i}}{\partial \mathbf{m}}\right) \\
\vdots \\
\left(\frac{\partial \widetilde{\Theta}_{n a}}{\partial \mathbf{m}}\right)
\end{array}\right]\right|_{j=\hat{j}}\left(\mathbf{m}^{*}-\widehat{\mathbf{m}}\right)+\xi
$$$$
=\boldsymbol{\Theta}_{j} \widetilde{\mathbf{j}}+\boldsymbol{\Theta}_{m} \widetilde{\mathbf{m}}+\boldsymbol{\xi}
$$$$
=\widetilde{\boldsymbol{\alpha}}^{T} \widetilde{\boldsymbol{\Theta}}+\widehat{\boldsymbol{\alpha}}^{T} \widetilde{\boldsymbol{\Theta}}+\widetilde{\boldsymbol{\alpha}}^{T} \widehat{\boldsymbol{\Theta}}+\Delta-u_{\mathrm{DC}},
$$

where $n_{a}$ is the number of nodes in the product layer and $\xi$ is a vector of higher-order terms. $\partial \widetilde{\Theta}_{i} / \partial \mathbf{j}$ and $\partial \widetilde{\Theta}_{i} / \partial \mathbf{m}$ are defined as

$$
\begin{gathered}
{\left[\frac{\partial \widetilde{\Theta}_{i}}{\partial \mathbf{j}}\right]^{T}=[\underbrace{0, \ldots, 0}_{(i-1) \times n_{k}}, \frac{\partial \widetilde{\Theta}_{i}}{\partial \mathbf{j}_{1 i}}, \ldots, \frac{\partial \widetilde{\Theta}_{i}}{\partial \mathbf{j}_{n_{k} i}}, \underbrace{0, \ldots, 0}_{\left(n_{a}-i\right) \times n_{k}}],} \\
{\left[\frac{\partial \widetilde{\Theta}_{i}}{\partial \mathbf{m}}\right]^{T}=[\underbrace{0, \ldots, 0}_{(i-1) \times n_{k}}, \frac{\partial \widetilde{\Theta}_{i}}{\partial \mathbf{m}_{1 i}}, \ldots, \frac{\partial \widetilde{\Theta}_{i}}{\partial \mathbf{m}_{n_{k} i}}, \underbrace{0, \ldots, 0}_{\left(n_{a}-i\right) \times n_{k}}] .}
\end{gathered}
$$

Substituting (18) into (17) yields

$$
\widetilde{u}=\widetilde{\boldsymbol{\alpha}}^{T} \widehat{\boldsymbol{\Theta}}+\widetilde{\mathbf{j}}^{T} \boldsymbol{\Theta}_{j}^{T} \widehat{\boldsymbol{\alpha}}+\widetilde{\mathbf{m}}^{T} \boldsymbol{\Theta}_{m}^{T} \widehat{\boldsymbol{\Theta}}+\Delta_{t}-u_{\mathrm{DC}} .
$$

Then, the derivative of $s(t)$ with (1) and (6) can be expressed as

$$
\begin{aligned}
\dot{s}(t) & =k_{1} \dot{e}_{1}(t)+\ddot{x}_{d}(t)-\ddot{x}(t)=k_{1} \dot{e}_{1}(t)+\ddot{x}_{d}(t) \\
- & {\left[f(\mathbf{x}, t)+g_{0} u(t)+d(t)\right]+g_{0} u_{\mathrm{IC}}^{*}(t)-g_{0} u_{\mathrm{IC}}^{*}(t) } \\
= & g_{0} u_{\mathrm{IC}}^{*}(t)-g_{0} u(t)+k_{1} \dot{e}_{1}(t)+\ddot{x}_{d}(t)-f(\mathbf{x}, t) \\
- & d(t)-\left[k_{1} \dot{e}_{1}(t)+\ddot{x}_{d}(t)-f(\mathbf{x}, t)-d(t)+e_{1}(t)\right. \\
+ & \left.k_{2} s(t)\right]=g_{0} \tilde{u}-e_{1}(t)-k_{2} s(t)=g_{0}\left(\widetilde{\boldsymbol{\alpha}}^{T} \widehat{\boldsymbol{\Theta}}\right. \\
+ & \left.\widetilde{\mathbf{j}}^{T} \boldsymbol{\Theta}_{j}^{T} \widehat{\boldsymbol{\alpha}}+\widetilde{\mathbf{m}}^{T} \boldsymbol{\Theta}_{m}^{T} \widehat{\boldsymbol{\Theta}}+\Delta_{t}-u_{\mathrm{DC}}\right)-e_{1}(t)-k_{2} s(t),
\end{aligned}
$$

where $\eta_{1}, \eta_{2}$, and $\eta_{3}$ are positive learning constants, $r$ is a prescribed attenuation constant. If the system starts with zero, $\mathrm{H} \infty$ tracking performance can be rewritten as

$$
\sup _{\Delta_{t}(t) \in L_{2}[0, T]} \frac{\int_{0}^{T} s^{2}(t) d t}{\int_{0}^{T} \Delta_{t}^{2}(t) d t} \leq \frac{r}{g_{0}} .
$$

To prove the stability of the DCFMWNN controller, define a Lyapunov function candidate in the following form [18]:

$$
\begin{aligned}
V_{2}(t)= & \frac{1}{2} e_{1}^{2}(t)+\frac{1}{2} s^{2}(t)+\frac{g_{0}}{2 \eta_{1}} \widetilde{\boldsymbol{\alpha}}^{T} \widetilde{\boldsymbol{\alpha}}+\frac{g_{0}}{2 \eta_{2}} \widetilde{\mathbf{j}}^{T} \widetilde{\mathbf{j}} \\
& +\frac{g_{0}}{2 \eta_{3}} \widetilde{\mathbf{m}}^{T} \widetilde{\mathbf{m}} .
\end{aligned}
$$

Taking the derivative of the Lyapunov function yields $\dot{V}_{2}(t)$

$$
\begin{aligned}
& =-k_{1} e_{1}^{2}(t)+s(t)\left[e_{1}(t)+\dot{s}(t)\right] \\
& -g_{0}\left(\frac{\widetilde{\boldsymbol{\alpha}}^{T} \dot{\hat{\boldsymbol{\alpha}}}}{\eta_{1}}+\frac{\tilde{\mathbf{j}}^{T} \dot{\hat{\mathbf{j}}}}{\eta_{2}}+\frac{\widetilde{\mathbf{m}}^{T} \dot{\hat{\mathbf{m}}}}{\eta_{3}}\right) \\
& =-k_{1} e_{1}^{2}(t)+e_{1}(t) s(t) \\
& -g_{0}\left(\frac{\widetilde{\boldsymbol{\alpha}}^{T} \dot{\hat{\boldsymbol{\alpha}}}}{\eta_{1}}+\frac{\tilde{\mathbf{j}}^{T} \dot{\mathbf{j}}}{\eta_{2}}+\frac{\widetilde{\mathbf{m}}^{T} \dot{\hat{\mathbf{m}}}}{\eta_{3}}\right) \\
& +s(t) g_{0}\left(\widetilde{\boldsymbol{\alpha}}^{T} \widehat{\boldsymbol{\Theta}}+\widetilde{\mathbf{j}}^{T} \boldsymbol{\Theta}_{j}^{T} \widehat{\boldsymbol{\alpha}}+\widetilde{\mathbf{m}}^{T} \boldsymbol{\Theta}_{m}^{T} \widehat{\boldsymbol{\alpha}}+\Delta_{t}-u_{\mathrm{DC}}\right) \\
& -s(t)\left(e_{1}(t)+k_{2} s(t)\right) \\
& \leq g_{0} \widetilde{\boldsymbol{\alpha}}^{T}\left[s(t) \widehat{\boldsymbol{\Theta}}-\frac{\dot{\hat{\boldsymbol{\alpha}}}}{\eta_{1}}\right]+g_{0} \widetilde{\mathbf{j}}^{T}\left[s(t) \boldsymbol{\Theta}_{j}^{T} \widehat{\boldsymbol{\alpha}}-\frac{\dot{\mathbf{j}}}{\eta_{2}}\right] \\
& +g_{0} \widetilde{\mathbf{m}}^{T}\left[s(t) \boldsymbol{\Theta}_{m}^{T} \widehat{\boldsymbol{\alpha}}-\frac{\dot{\hat{\mathbf{m}}}}{\eta_{3}}\right]+g_{0} s(t)\left[\Delta_{t}-u_{\mathrm{DC}}\right] \text {. }
\end{aligned}
$$

In order to further simplify $\dot{V}_{2}(t)$, the adaptive learning laws must be guaranteed as follows:

$$
\begin{aligned}
& \dot{\hat{\boldsymbol{\alpha}}}=\eta_{1} s(t) \widehat{\boldsymbol{\Theta}}, \\
& \dot{\hat{\mathbf{j}}}=\eta_{2} s(t) \boldsymbol{\Theta}_{j}^{T} \widehat{\boldsymbol{\alpha}},
\end{aligned}
$$




$$
\begin{aligned}
\dot{\dot{\mathbf{m}}} & =\eta_{3} s(t) \Theta_{m}^{T} \widehat{\boldsymbol{\alpha}}, \\
u_{\mathrm{DC}} & =\frac{r^{2}+1}{2 r^{2}} s(t),
\end{aligned}
$$

where $\eta_{1}$ plays a great effect on the coefficients of $\varphi_{j, m}$ and $\psi_{j, m}$ and the value of $\eta_{1}$ is set to be too small to enhance the influences of $\varphi_{j, m}$ and $\psi_{j, m}$, which leads to the low convergence rate of the control system. $\eta_{2}$ and $\eta_{3}$ are related to the dilation and translation parameters, which are set to be too big or small to achieve the high convergence rate of the control system. $r$ is used in the DC controller; the output of $\mathrm{DC}$ is too little to reduce the approximation error when the value of $r$ is set to be more than 2. However, the value of $r$ is set to be too small; there exists an obvious overshoot.

Thus, substituting (26) into (25) yields the following:

$$
\begin{aligned}
\dot{V}_{2}(t) & \leq g_{0} s(t)\left[\Delta_{t}-\frac{r^{2}+1}{2 r^{2}} s(t)\right] \\
& \leq-\frac{1}{2} g_{0}^{2} s^{2}(t)-\frac{1}{2}\left[\frac{g_{0} s(t)}{r}-r \Delta_{t}\right]^{2}+\frac{1}{2} r^{2} \Delta_{t}^{2} \\
& \leq-\frac{1}{2} g_{0}^{2} s^{2}(t)+\frac{1}{2} r^{2} \Delta_{t}^{2} .
\end{aligned}
$$

Assume $\Delta_{t}(t) \in L_{2}[0, T]$ and $\forall T \in[0, \infty)$. Integrating the above equation from $t=0$ to $t=T$ yields

$$
V_{2}(T)-V_{2}(0) \leq-\frac{g_{0}^{2}}{2} \int_{0}^{T} s^{2}(t) d t+\frac{r^{2}}{2} \int_{0}^{T} \Delta_{t}^{2} d t
$$

Since $V_{2}(T)$ is positive semidefinite, the above inequality is as follows:

$$
\frac{g_{0}^{2}}{2} \int_{0}^{T} s^{2}(t) d t \leq V_{2}(0)+\frac{r^{2}}{2} \int_{0}^{T} \Delta_{t}^{2} d t
$$

In combination with (23), the following inequality can be obtained:

$$
\begin{aligned}
\frac{g_{0}^{2}}{2} \int_{0}^{T} s^{2}(t) d t \leq & \frac{e_{1}^{2}(0)+s^{2}(0)}{2}+\frac{g_{0} \widetilde{\boldsymbol{\alpha}}^{T}(0) \widetilde{\boldsymbol{\alpha}}(0)}{2 \eta_{1}} \\
& +\frac{g_{0} \widetilde{\mathbf{j}}^{T}(0) \widetilde{\mathbf{j}}(0)}{2 \eta_{2}}+\frac{g_{0} \widetilde{\mathbf{m}}^{T}(0) \widetilde{\mathbf{m}}(0)}{2 \eta_{3}} \\
& +\frac{r^{2}}{2} \int_{0}^{T} \Delta_{t}^{2}(t) d t .
\end{aligned}
$$

As a result, the stability of the control system can be guaranteed.

\section{Semiphysical Simulation}

In order to demonstrate the feasibility and effectiveness of the DCFMWNN controller, the designed reference commands, with different algorithms, like the convergence analysis of the MWNN and FMWNN, the step response of the
TABLE 1: The parameters of the torque motor.

\begin{tabular}{lc}
\hline Parameters & Values \\
\hline Rated power $p_{r}$ & $2.8 \mathrm{kw}$ \\
Biggest locked-rotor torque $T_{\text {max }}$ & $19.8 \mathrm{~N}$ \\
Continuous locked-rotor torque $T_{\text {cont }}$ & $12 \mathrm{~N}$ \\
Continuous current $I_{\text {cont }}$ & $80 \mathrm{~A}$ \\
$\begin{array}{l}\text { Rated speed } v_{r} \\
\text { Coefficient of electromagnetic } \\
\text { torque } K_{t}\end{array}$ & $1200 \mathrm{r} / \mathrm{min}$ \\
$\begin{array}{l}\text { Coefficient of counter } \\
\text { electromotive force } K_{e}\end{array}$ & $2.6 \mathrm{~N} \cdot \mathrm{m} / \mathrm{A}$ \\
$\begin{array}{l}\text { Equivalent inductance } \\
\text { of armature circuit } L_{m}\end{array}$ & $9.6 \mathrm{~V} / \mathrm{krpm}$ \\
$\begin{array}{l}\text { Equivalent resistance } \\
\text { of armature circuit } R_{m}\end{array}$ & $0.0036 \mathrm{H}$ \\
$\begin{array}{l}\text { Rotational inertia } J_{m} \\
\text { Viscous friction coefficient } B_{m}\end{array}$ & $1.2 \Omega$ \\
\hline
\end{tabular}

FMWNN and DCFMWNN, and the sinusoidal tracking of the FMWNN and DCFMWNN, are applied to the simulation platform of the ELS shown in Figure 3. The host control computer with the proposed algorithm to achieve torque tracking control of the ELS mainly analyses and calculates the feedback torque of the torque sensor, the compensation torque of inertia disks, and the actual torque required. The signal conditioning center is mainly used for processing signals from the sensors and the configuration settings needed for the hardware switches. According to the output of the host control computer, the output of drive provides the appropriate current to drive the torque motor. The torque sensor is used to collect the output of the torque motor, which is fed back to the host control computer to form the closed-loop control for the loading torque. The function of the resolver is gathering the angle position of the gear reducer, which can be used to calculate the real-time command torque for the torque motor. The function of the angular velocity sensor is gathering the angular velocity of the gear reducer to provide more actual values for the identification methods and the control strategies. The angular acceleration sensor collects the angular acceleration at the end of the gear reducer to compute the inertia moment produced by the inertia disks. The number of inertia disks depends on the actual demand of the gun control system, which provides various rotational inertia values to simulate the inertia torque.

The following simulations are carried out in an Intel Core i5 CPU with $3.2 \mathrm{GHz}$ rate, $4 \mathrm{~GB}$ RAM, and 64-bit operating system. The parameters of the actual gun control system are as follows: the rotary inertia of the turret is $7000 \mathrm{~kg} \cdot \mathrm{m}^{2}$, the total friction torque $M_{f}$ is less than $1200 \mathrm{~N} \cdot \mathrm{m}$, the center position of gravity is located in $0.35 \mathrm{~m}$ away from the rotation center of the turret, and the reduction ratio is 360 . The controller parameters are selected as $\eta_{1}=12, \eta_{2}=\eta_{3}=0.1$, and $r=$ 0.5 . By analysing the simulation results, the control period is $5 \mathrm{~ms}$ and the sampling frequency is $10 \mathrm{kHz}$, and the important torque motor parameters are shown in Table 1 . 


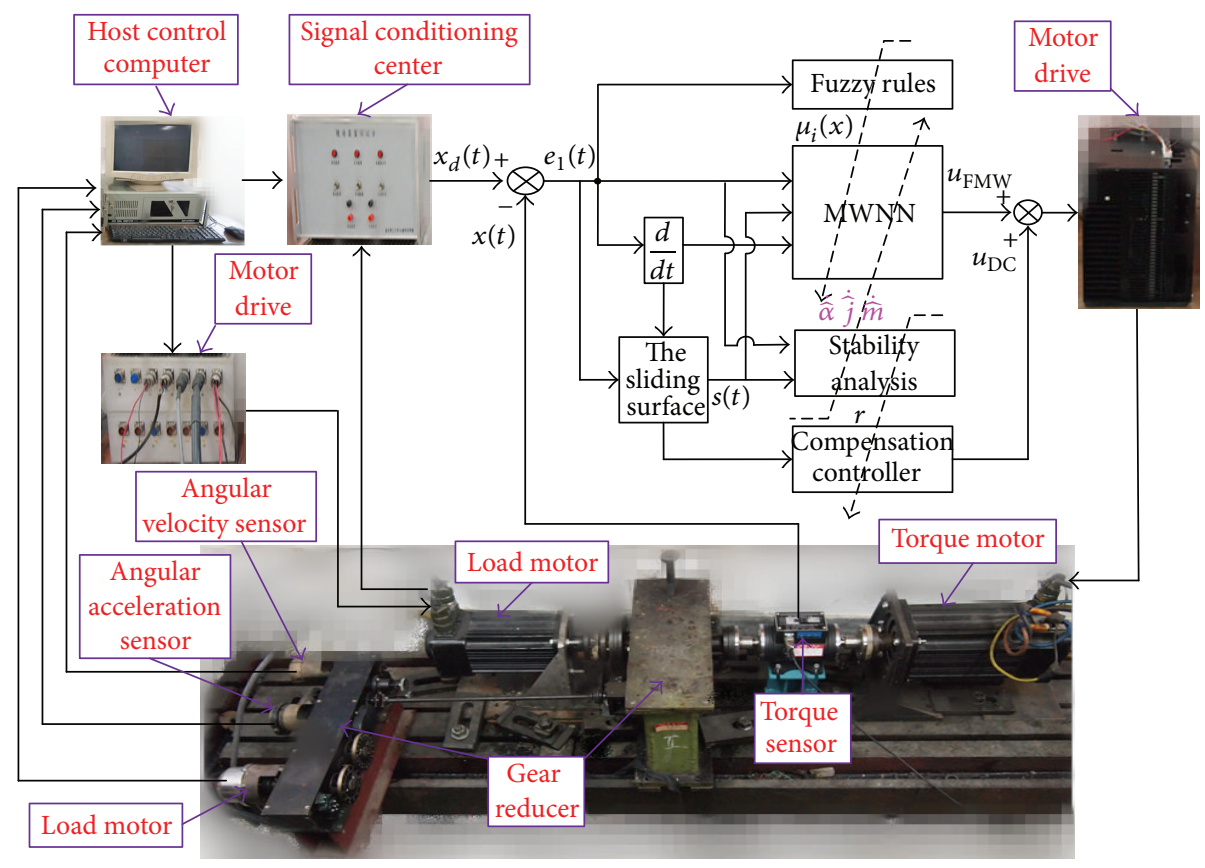

FIGURE 3: The simulation platform of the ELS.

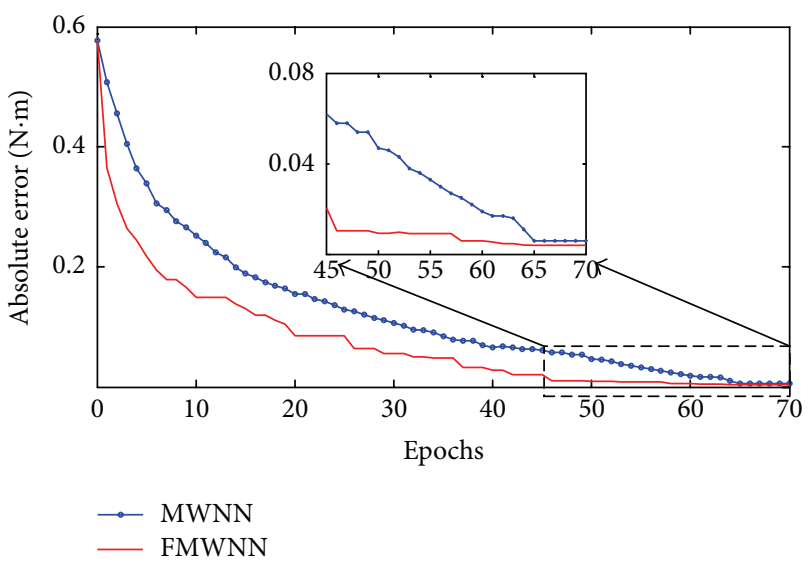

FIGURE 4: A comparison of the convergence epochs for the MWNN and FMWNN controller.

4.1. Convergence Analysis. The superiority between the proposed MWNN and FMWNN controller is illustrated by analysing the convergence epochs, so the absolute error $\left(\left|x_{d}(t)-x(t)\right|\right)$ of the training data is adopted to evaluate the MWNN and FMWNN controller. The simulation results are shown in Figure 4, which show that the convergence epochs of the MWNN and FMWNN are 65 and 46 when the absolute error is close to $0.01 \mathrm{~N} \cdot \mathrm{m}$. In general, a larger absolute error is set, which contributes to the acceleration of the convergence process. However, if a higher precision needs to be guaranteed, the absolute error must be set to be smaller comparatively. Meanwhile, the convergence process will be slower because of the computing load brought.

4.2. Step Response. The step response with external disturbance is evaluated in Figure 5, which further indicates the

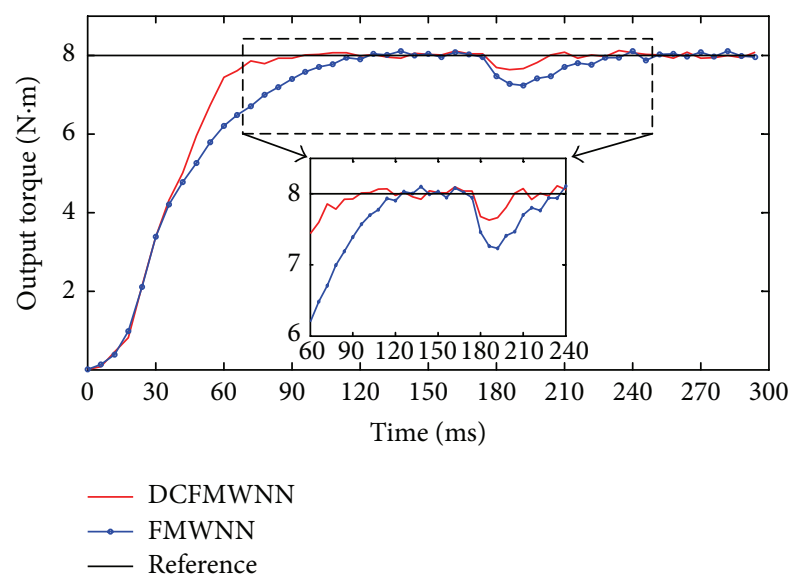

FIGURE 5: A comparison of step response with external disturbance.

rapidity and stability of the FMWNN and DCFMWNN controller in the ELS. From Figure 5, it is clear that the FMWNN controller is $30 \mathrm{~ms}$ slower than the DCFMWNN when the step response of the reference command is set to be $8 \mathrm{~N} \cdot \mathrm{m}$ at the initial stage. At the time of $170 \mathrm{~ms}$, an external disturbance $-2 \mathrm{~N} \cdot \mathrm{m}$ lasting $5 \mathrm{~ms}$ is added to the control system; the latter performs better than the former, which proves that the addition of DC improves the robustness of the control system to some extent.

4.3. Sinusoidal Tracking. In order to compare the superiority of the FMWNN and DCFMWNN, the reference command of the torque motor is usually chosen as $x_{d 1}(t)=5 \sin 2 \pi t$ in view of practical working conditions, when the load motor is working in sinusoidal position motion $\Delta$ dit $=\sin (5 \pi t) \mathrm{rad}$. 
TABLE 2: The dynamic simulation results of the sinusoidal tracking.

\begin{tabular}{|c|c|c|c|c|}
\hline \multirow{2}{*}{ The desired reference command } & \multicolumn{2}{|c|}{ Mean amplitude error (\%) } & \multicolumn{2}{|c|}{ Mean phase error $\left({ }^{\circ}\right)$} \\
\hline & FMWNN & DCFMWNN & FMWNN & DCFMWNN \\
\hline $5 \sin 20 \pi t$ & 9.45 & 6.82 & -9.6325 & -9.0483 \\
\hline $5 \sin 10 \pi t$ & 7.28 & 5.76 & -7.0729 & -5.2541 \\
\hline $\sin 2 \pi t$ & 6.51 & 4.25 & -5.3263 & -4.1036 \\
\hline $5 \sin 2 \pi t$ & 5.36 & 3.53 & -3.2541 & -2.0527 \\
\hline $10 \sin 2 \pi t$ & 4.12 & 2.53 & -2.2692 & -0.9235 \\
\hline
\end{tabular}

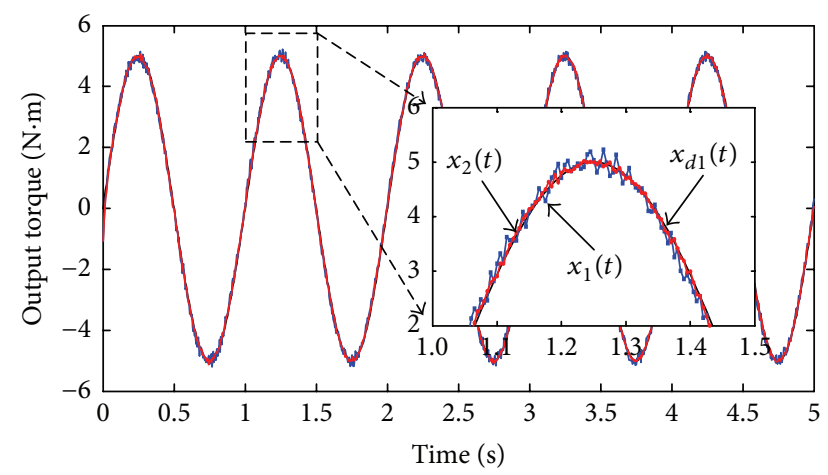

Figure 6: The sinusoidal tracking with $x_{d 1}(t)=5 \sin 2 \pi t$.

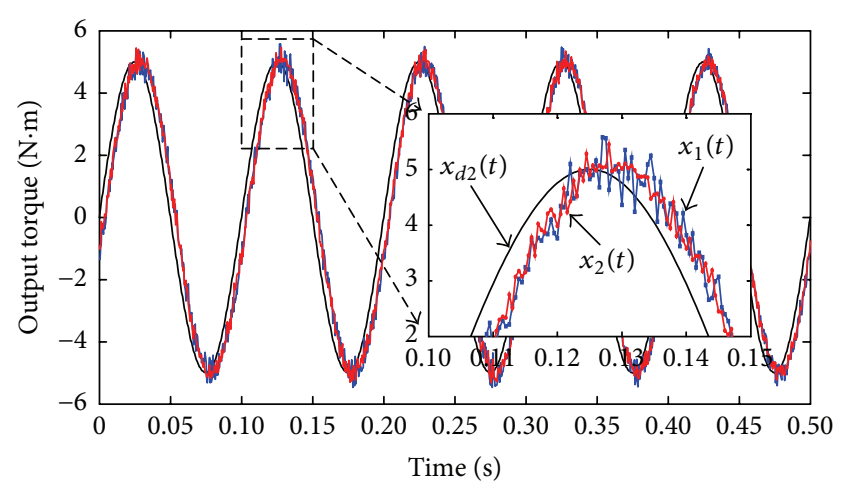

Figure 7: The sinusoidal tracking with $x_{d 2}(t)=5 \sin 20 \pi t$.

The outputs of the FMWNN and DCFMWNN controller are $x_{1}(t)$ and $x_{2}(t)$ shown in Figure 6.

Figure 6 once again proves that the performance of the DCFMWNN controller is better than the FMWNN controller from the mean tracking errors of the amplitude and phase. The mean tracking error of the FMWNN even exceeds $9 \%$, which is far greater than the DCFMWNN.

In order to further analyse the stability and precision of the desired outputs with different frequencies and amplitude, the reference commands of the torque motor are selected as $x_{d 2}(t)=5 \sin 20 \pi t, x_{d 3}(t)=5 \sin 10 \pi t, x_{d 4}(t)=\sin 2 \pi t$, and $x_{d 5}(t)=10 \sin 2 \pi t$, and the simulation results are presented in Figures 7-10, respectively. The dynamic simulation results of the sinusoidal tracking are shown in detail in Table 2, in

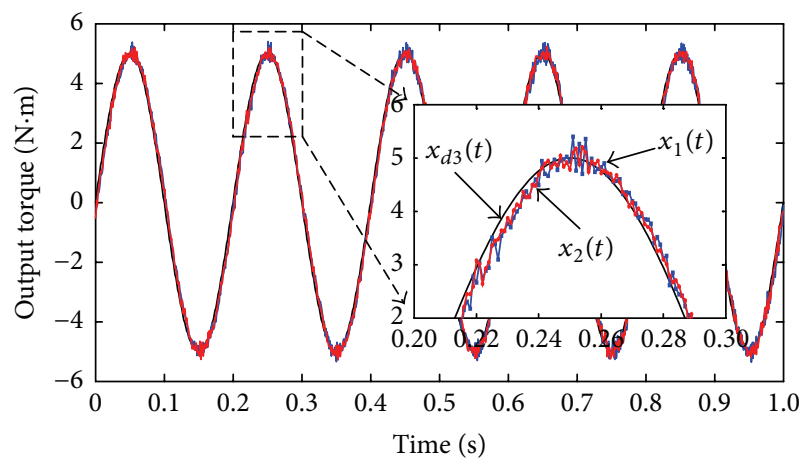

FIgURE 8: The sinusoidal tracking with $x_{d 3}(t)=5 \sin 10 \pi t$.

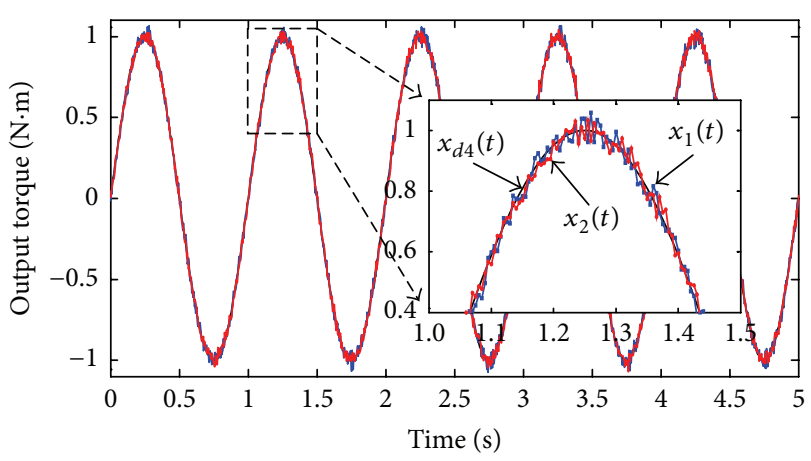

FIGURE 9: The sinusoidal tracking with $x_{d 4}(t)=\sin 2 \pi t$.

which the negative values of the mean phase error mean that the real outputs lag behind the reference command. Consider

Mean amplitude error

$$
=\frac{\sum_{i=1}^{N_{\mathrm{me}}}\left|x(i)-x_{d}(i)\right| /|x(i)|}{N_{\mathrm{me}}} \times 100 \%,
$$

$$
\text { Mean phase error }=\frac{\sum_{i=1}^{N_{\mathrm{me}}}\left(\theta(i)-\theta_{d}(i)\right)}{N_{\mathrm{me}}},
$$

where $N_{\text {me }}$ is the sum number of the output in certain control time; $x(i)$ and $x_{d}(i)$ are the real and ideal output amplitude; $\theta(i)$ and $\theta_{d}(i)$ are the real and ideal output phase.

The mean amplitude and mean phase errors are the mean absolute values and angle deviations between the desired 


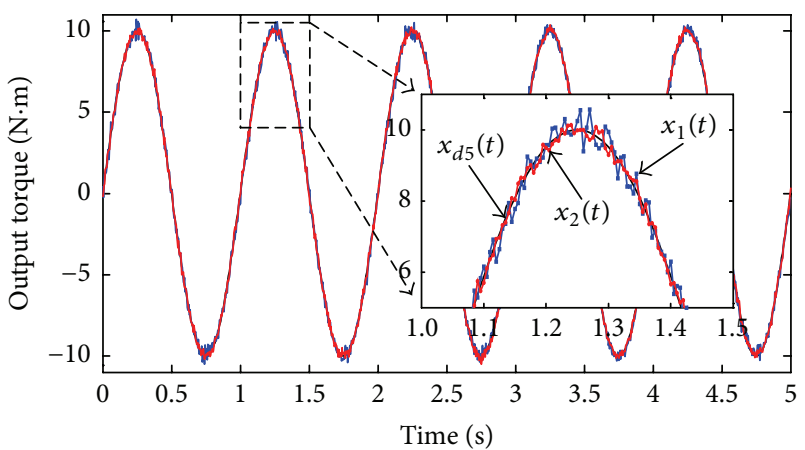

FIGURE 10: The sinusoidal tracking with $x_{d 5}(t)=10 \sin 2 \pi t$.

reference command and the real simulation outputs, which show that the smaller the reference command amplitudes and the higher the reference command frequencies (within certain limits), the worse the control performance. However, what we need is to remember that the control effectiveness is relatively poorer when the direction of the torque motor is changed, which plays a negative effect on the overall performance and needs to be delved into in the future.

\section{Conclusion}

This paper has successfully investigated the ELS via DCFMWNN controller on the simulation platform of the ELS. The FMWNN controller has salient merits of free model, effective search, and computation ability with few chattering phenomena, and the DC controller is introduced to eliminate the effect of the approximation error and guarantee the system stability in the sense of Lyapunov theory. Furthermore, the simulation results are promising and reveal that the proposed controller is able to successfully damp the interarea nonlinearities and maintain system precision and robustness.

\section{Conflict of Interests}

The authors declare that there is no conflict of interests regarding the publication of this paper.

\section{Acknowledgments}

The authors are grateful to the reviewers for their valuable comments. The authors appreciate the partial financial support from the National Science Foundation of China under Grant 51305205.

\section{References}

[1] Z. X. Jiao, J. X. Gao, Q. Hua, and S.-P. Wang, "The velocity synchronizing control on the electro-hydraulic load simulator," Chinese Journal of Aeronautics, vol. 17, no. 1, pp. 39-46, 2004.

[2] Y. X. Shang, H. Yuan, Z. X. Jiao, and N. Yao, "Matching design of hydraulic load simulator with aerocraft actuator," Chinese Journal of Aeronautics, vol. 26, no. 2, pp. 470-480, 2013.
[3] J. Y. Yao, Z. X. Jiao, Y. X. Shang, and C. Huang, "Adaptive nonlinear optimal compensation control for electro-hydraulic load simulator," Chinese Journal of Aeronautics, vol. 23, no. 2, pp. 720-733, 2010.

[4] J. Y. Yao, Z. X. Jiao, and S. S. Han, "Friction compensation for low velocity control of hydraulic flight motion simulator: a simple adaptive robust approach," Chinese Journal of Aeronautics, vol. 26, no. 3, pp. 814-822, 2013.

[5] J. Yao, Z. Jiao, and B. Yao, "Robust control for static loading of electro-hydraulic load simulator with friction compensation," Chinese Journal of Aeronautics, vol. 25, no. 6, pp. 954-962, 2012.

[6] C. Otto and H. Petr, "Adaptive fuzzy sliding mode control for electro-hydraulic servo mechanism," Expert Systems with Applications, vol. 39, no. 11, pp. 10269-10277, 2012.

[7] C. W. Wang, Z. X. Jiao, S. Wu, and Y. X. Shang, "Nonlinear adaptive torque control of electro-hydraulic load system with external active motion disturbance," Mechatronics, vol. 24, no. 1, pp. 32-40, 2014.

[8] C. C. Tsai, Y. Y. Li, F. C. Tai, and C. H. Lu, "Intelligent adaptive motion control using fuzzy basis function networks for electric unicycle," Asian Journal of Control, vol. 17, no. 3, pp. 977-993, 2015.

[9] W. X. Fu, L. Sun, Y. F. Yu, S. P. Zhu, and J. Yan, "Design and model-building of motor-driven load simulator with large torque outputs," Journal of System Simulation, vol. 21, no. 12, pp. 3596-3598, 2009.

[10] C. C. Tsai, Z. C. Wang, C. T. Lee, and Y. Y. Li, "Intelligent adaptive trajectory tracking control for an autonomous smallscale helicopter using fuzzy basis function networks," Asian Journal of Control, vol. 17, no. 1, pp. 234-245, 2015.

[11] B. Guo, The System Research of Electric Load Simulator Control, Harbin Institute of Technology, Harbin, China, 2012.

[12] D. K. Shen, Q. Hua, and Z. L. Wang, "Motor-driven load system based on neural networks," Acta Aeronautica et Astronautica Sinica, vol. 23, no. 6, pp. 525-529, 2002.

[13] K. S. Narendra and K. Parthasarathy, "Identification and control of dynamical systems using neural networks," IEEE Transactions on Neural Networks, vol. 1, no. 1, pp. 4-27, 1990.

[14] C. Elmas, O. Ustun, and H. H. Sayan, "A neuro-fuzzy controller for speed control of a permanent magnet synchronous motor drive," Expert Systems with Applications, vol. 34, no. 1, pp. 657664, 2008.

[15] C. E. Castañeda and P. Esquivel, "Decentralized neural identifier and control for nonlinear systems based on extended Kalman filter," Neural Networks, vol. 31, pp. 81-87, 2012.

[16] J. M. Deng, "Dynamic neural networks with hybrid structures for nonlinear system identification," Engineering Applications of Artificial Intelligence, vol. 26, no. 1, pp. 281-292, 2013.

[17] J. Kang, W. J. Meng, A. Abraham, and H. B. Liu, "An adaptive PID neural network for complex nonlinear system control," Neurocomputing, vol. 135, pp. 79-85, 2014.

[18] C. F. Hsu, "Adaptive PI Hermite neural control for MIMO uncertain nonlinear systems," Applied Soft Computing Journal, vol. 13, no. 5, pp. 2569-2576, 2013.

[19] Q. H. Zhang and A. Benveniste, "Wavelet networks," IEEE Transactions on Neural Networks, vol. 3, no. 6, pp. 889-898, 1992.

[20] J. Zhang, G. G. Walter, Y. B. Miao, and W. N. W. Lee, "Wavelet neural networks for function learning," IEEE Transactions on Signal Processing, vol. 43, no. 6, pp. 1485-1497, 1995.

[21] B. R. Bakshi and G. Stephanopoulos, "Wave-net: a multiresolution, hierarchical neural network with localized learning," AIChE Journal, vol. 39, no. 1, pp. 57-81, 1993. 
[22] H. L. Wei and S. A. Billings, "Identification of time-varying systems using multiresolution wavelet models," International Journal of Systems Science, vol. 33, no. 15, pp. 1217-1228, 2002.

[23] C. C. Chang and Y. F. Shi, "Identification of time-varying hysteretic structures using wavelet multiresolution analysis," International Journal of Non-Linear Mechanics, vol. 45, no. 1, pp. 21-34, 2010.

[24] K. Premkumar and B. V. Manikandan, "Adaptive Neuro-Fuzzy Inference System based speed controller for brushless DC motor," Neurocomputing, vol. 138, pp. 260-270, 2014.

[25] A. Ebadat, N. Noroozi, A. A. Safavi, and S. H. Mousavi, "New fuzzy wavelet network for modeling and control: the modeling approach," Communications in Nonlinear Science and Numerical Simulation, vol. 16, no. 8, pp. 3385-3396, 2011.

[26] M. S. Kahkeshi, F. Sheikholeslam, and M. Zekri, "Design of adaptive fuzzy wavelet neural sliding mode controller for uncertain nonlinear systems," ISA Transactions, vol. 52, no. 3, pp. 342-350, 2013.

[27] M. Alizadeh and M. Tofighi, "Full-adaptive THEN-part equipped fuzzy wavelet neural controller design of FACTS devices to suppress inter-area oscillations," Neurocomputing, vol. 118, no. 2, pp. 157-170, 2013.

[28] M. Wu and J. S. Chen, "A discrete-time global quasi-sliding mode control scheme with bounded external disturbance rejection," Asian Journal of Control, vol. 16, no. 6, pp. 1839-1848, 2014.

[29] R. J. Lian, "Design of an enhanced adaptive self-organizing fuzzy sliding-mode controller for robotic systems," Expert Systems with Applications, vol. 39, no. 1, pp. 1545-1554, 2012.

[30] P. Li and F.-J. Jin, "Adaptive fuzzy control for unknown nonlinear systems with perturbed dead-zone inputs," Acta Automatica Sinica, vol. 36, no. 4, pp. 573-579, 2010.

[31] J. Matuško, Š. Ileš, F. Kolonić, and V. Leši, "Control of 3D tower crane based on tensor product model transformation with neural friction compensation," Asian Journal of Control, vol. 17, no. 2, pp. 443-458, 2015.

[32] X. Liu and X. C. Dong, "4-channel sliding mode vontrol of teleoperation systems with disturbances," Asian Journal of Control, vol. 17, no. 4, pp. 1267-1273, 2015.

[33] I. B. Tijani and R. Akmeliawati, "Support vector regression based friction modeling and compensation in motion control system," Engineering Applications of Artificial Intelligence, vol. 25, no. 5, pp. 1043-1052, 2012.

[34] F. M. Fayez, "Adaptive hybrid control system using a recurrent RBFN-based self-evolving fuzzy-neural-network for PMSM servo drives," Applied Soft Computing Journal, vol. 21, no. 2, pp. 509-532, 2014.

[35] Q. Gao, Z. Sun, G. L. Yang, R. Hou, L. Wang, and Y. L. Hou, "A novel active disturbance rejection-based control strategy for a gun control system," Journal of Mechanical Science and Technology, vol. 26, no. 12, pp. 4141-4148, 2012.

[36] J. J. E. Slotine and W. P. Li, Applied Nonlinear Control, PrenticeHall, Englewood Cliffs, NJ, USA, 1991.

[37] T. Takagi and M. Sugeno, "Fuzzy identification of systems and its applications to modeling and control," IEEE Transactions on Systems, Man and Cybernetics, vol. 15, no. 1, pp. 116-132, 1985.

[38] B. Beliczynski, "A method of multivariable Hermite basis function approximation," Neurocomputing, vol. 96, no. 1, pp. 1218, 2012.

[39] L. X. Wang, Adaptive Fuzzy Systems and Control: Design and Stability Analysis, Prentice-Hall, Englewood Cliffs, NJ, USA, 1994.
[40] B. S. Chen, C. H. Lee, and Y. C. Chang, "Ho tracking design of uncertain nonlinear SISO systems: adaptive fuzzy approach," IEEE Transactions on Fuzzy Systems, vol. 4, no. 1, pp. 32-43, 1996. 


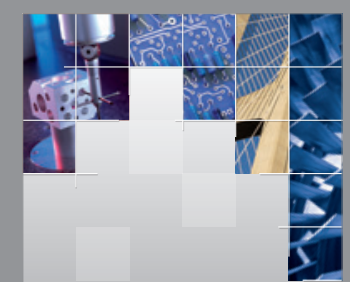

\section{Enfincering}
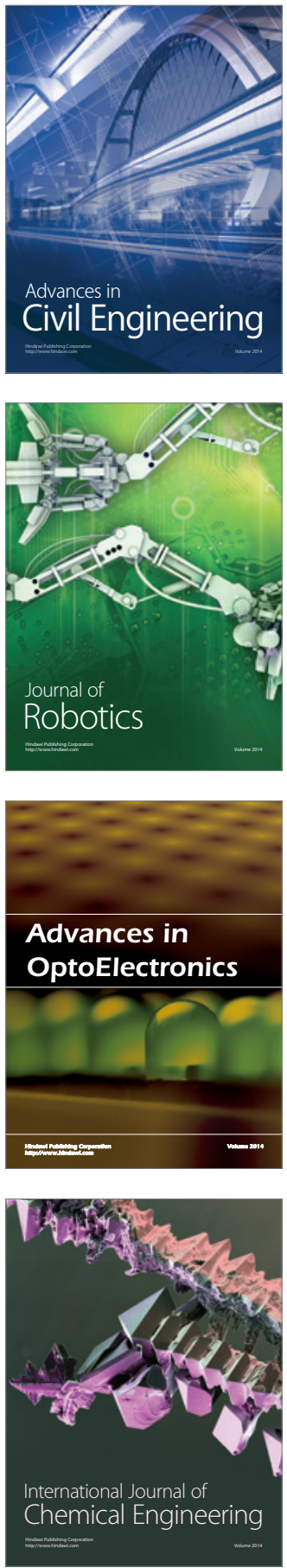

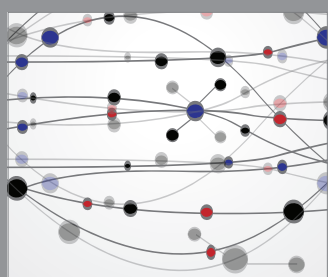

The Scientific World Journal

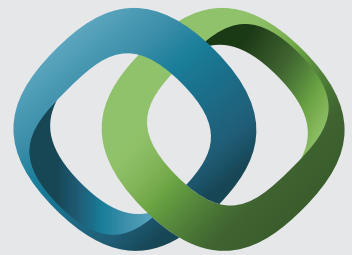

\section{Hindawi}

Submit your manuscripts at

http://www.hindawi.com
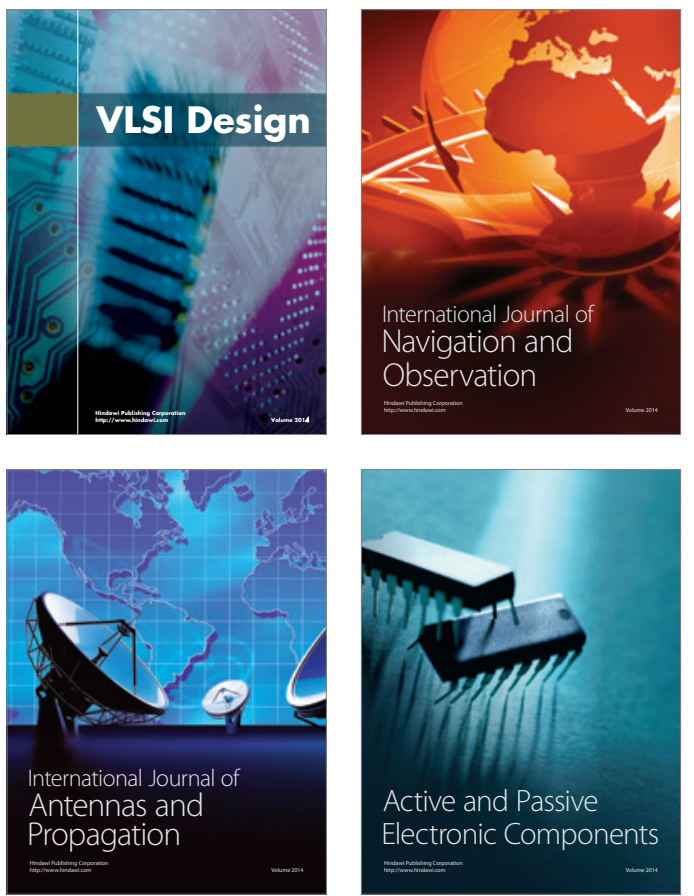
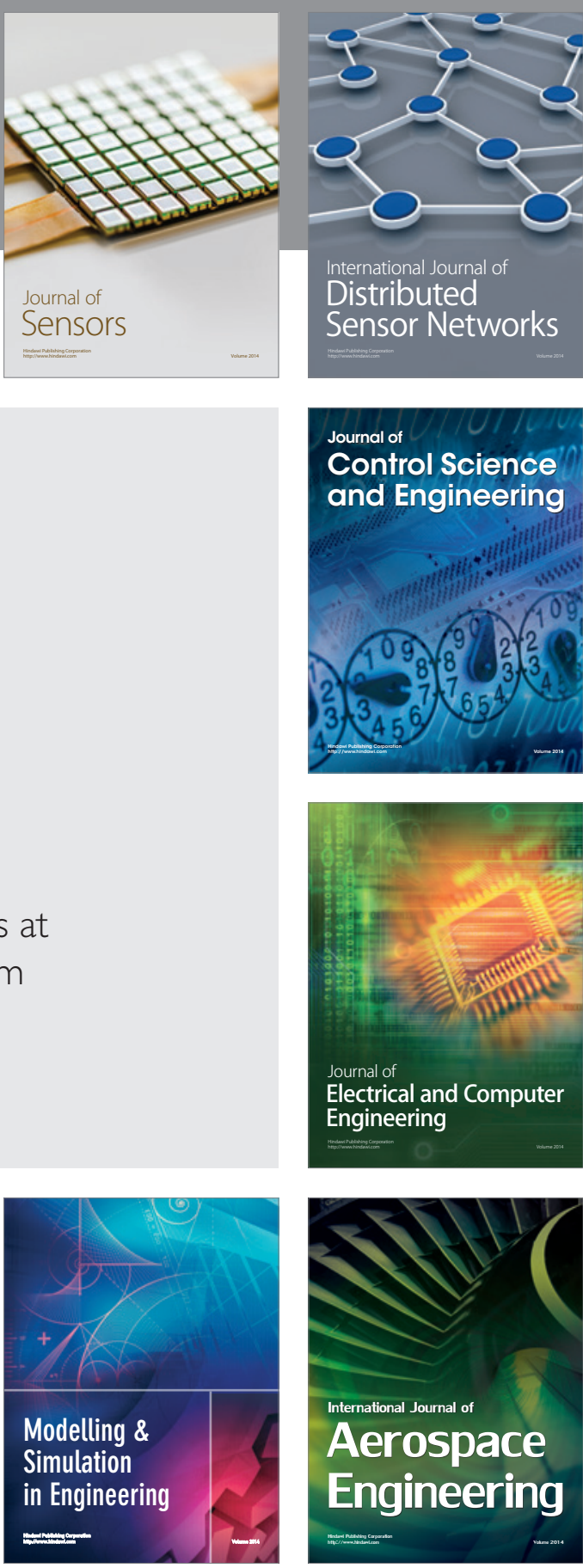

International Journal of

Distributed

Sensor Networks

Journal of

Control Science

and Engineering
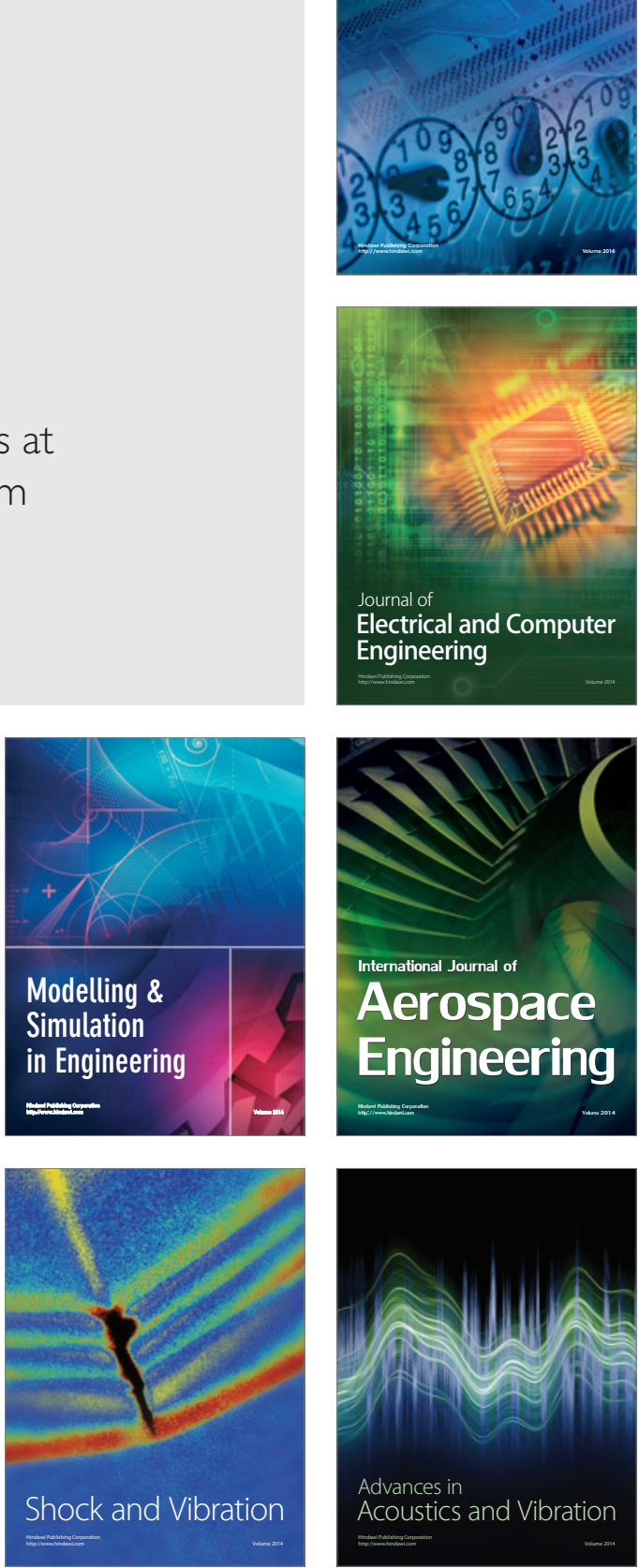